\title{
CONFRONTATION DU RÉEL ET DU VIRTUEL LA CONSTRUCTION D'UNE RELATION EN FORMATION À DISTANCE
}

\author{
Serge de Patoul ${ }^{1}$
}

\section{Avant-propos}

Cet article est une adaptation d'une leçon publique donnée à l'occasion de la présentation d'une dissertation doctorale ${ }^{2}$ intitulée Réelle distance ou distance virtuelle, communication virtuelle ou réelle communication? Effets sur le processus d'apprentissage des apprenants à distance, de la présence d'étudiants sur le document audiovisuel de formation. Contribution à l'étude de la transmission des savoirs.

Nous allons contextualiser la recherche, présenter le raisonnement et brièvement l'expérience et les résultats. Nous terminerons en traçant des pistes pour de nouvelles recherches.

Précisons une optique fondamentale choisie délibérément dès le début de la recherche : travailler sur base d'une expérimentation de type laboratoire.

1 Maître de conférence à l'Université catholique de Louvain.

2 Thèse de doctorat, Université catholique de Louvain, Louvain-la-Neuve, février 1999.

Recherches en communication, $\mathrm{n}^{\circ} 13,(2000)$. 


\section{La contextualisation de la recherche}

Aujourd'hui, dans notre monde occidental, la rentabilité de l'acte est de plus en plus prise en référence. Partons d'un raisonnement économique, d'une logique de gestionnaire. Dans le secteur de l'enseignement, un constat peut être énoncé : il y a une énorme perte d'énergie. Un cours se fait et il passe. Au moment où il se donne, il s'envole. Chaque année de nombreux cours se répètent ou plutôt s'enseignent de façon très similaire aux années précédentes. Dès lors, une question simple se pose : comment minimiser cette perte d'énergie ? Dans une démarche économique de production de valeur, la réponse est la création de supports de formation qui diminuent l'intervention de l'enseignant, qui gardent en mémoire ce qui, au cours, "s'envole" pour "l'exploiter" à plusieurs reprises. Ceci mène donc à la mise sur support durable d'un cours. Cette pratique de l'enseignement est celle de la formation à distance.

Sur base d'une observation de pratique de formation à distance ainsi que sur base de la littérature, trois remarques doivent être mises en évidence.

Une première observation de la formation à distance montre que cette forme d'enseignement se gère suivant des principes de gestion d'entreprise et plus précisément de gestion industrielle : calcul de rentabilité de l'investissement, approche de marché sur base des demandes potentielles, gestion de la production, gestion des stocks ... Dans cette perspective, il est intéressant de citer une situation très révélatrice. Sans que ce soit énoncé aussi explicitement, force est de constater qu'une institution de formation à distance est fortement tentée de maintenir l'utilisation d'un cours à distance même si certains aspects du cours sont devenus obsolètes, quitte à intégrer des errata. La raison est la nécessité d'arriver à écouler un nombre minimum de cours, d'atteindre le point mort qui évite les pertes et de liquider les stocks. Au vu de cette observation, minimiser le coût d'investissement afin d'atteindre le plus rapidement ce fameux point mort est un objectif à se fixer parce que cela représente une garantie d'avoir des cours plus actuels. En d'autres termes, arriver à une formule peu onéreuse, créatrice de peu de stocks assure un meilleur renouvellement des cours.

La seconde observation liée à la formation à distance est le nombre d'étudiants qui abandonnent. Un débat sur les raisons et les 
statistiques des abandons est opportun ${ }^{1}$. Dans cet article, nous nous limitons à constater que la cause essentiellement citée est le problème de l'isolement de l'apprenant à distance. La difficulté de la formation à distance se situe dans la rupture de la communication. Nous pouvons définir la formation à distance comme étant une formation où l'acte d'enseigner et l'acte d'apprentissage ne se font pas en même temps. Notons que la distance peut être de trois types : une distance physique ou géographique, une distance temporelle et une distance psychologique.

Ajoutons une troisième observation générale sur les besoins de formations. Plusieurs études prospectives sur les besoins de formation développent l'idée que le savoir augmente de plus en plus vite. Dès lors, un travailleur a l'obligation de se "reformer" à plusieurs reprises durant sa carrière professionnelle. Ce besoin de formation apparaît comme de plus en plus évident au vu du rythme accéléré de l'augmentation du savoir. C'est ainsi que dans son projet pédagogique, l'université ${ }^{2}$ définit de plus en plus le rôle de l'enseignant comme celui qui doit donner le goût à apprendre et la capacité d'accéder à la connaissance 3 .

1 Toutes les statistiques montrent qu'il s'agit d'un point noir de ce type de formation. Il y a lieu de corriger cette première perception. Effectivement, les informations se résument à constater que l'apprenant à distance ne fait plus signe de vie c'est-à-dire qu'il n'effectue plus les travaux demandés par l'institution de formation ou ne présente pas les tests prévus. Si l'on considère l'étudiant adulte en formation à distance comme autonome, il a la capacité de définir ses besoins de formation. Dès lors, les statistiques d'abandon doivent être affinées pour distinguer la situation de réel abandon de celle où l'apprenant considère avoir eu l'apprentissage qui lui était nécessaire. En d'autres termes, l'adulte capable de gérer sa formation et ses apprentissages va se comporter face au programme de formation ou à un cours comme son propre "évaluateur". Il jugera si son besoin de formation est satisfait ou si la formation répond à son besoin. Il appréciera ce qui lui apparaît nécessaire d'effectuer. Il s'auto-évalue avec évidemment le risque d'erreur qui incombe à cette forme d'appréciation des apprentissages. Il y a donc dans les statistiques d'abandons officielles de réels abandons et des apprenants autonomes qui, ayant acquis ce qu'ils considèrent comme devant être acquis, ont arrêté de suivre la formation. Une question qui peut dès lors se poser est de savoir s'il y a une corrélation entre le degré d'autonomie et le résultat de la formation à distance sachant que le résultat doit être compris comme le fait d'atteindre l'objectif de formation que l'apprenant s'est fixé préalablement.

2 C'est l'institution universitaire en général qui est prise en considération. En particulier, nous pouvons citer le projet pédagogique de l'Université catholique de Louvain intitulé "Gérer sa formation".

3 Une des conclusions d'un colloque tenu en mai 1998 à Glion (Suisse) réunissant une vingtaine de personnalités du monde de l'éducation supérieure reprise par 


\section{Le raisonnement suivi}

Dans ce contexte, une option pour la recherche a été prise : aborder la formation à distance pour adulte, plus précisément pour des apprenants ayant au moins un diplôme d'humanités secondaires supérieures.

Ayant fait ce choix de travailler la formation à distance pour adultes, un aspect pédagogique devient essentiel. L'apprenant est une personne qui possède déjà bien des connaissances et en particulier des connaissances sur le système classique d'enseignement. Il en a donc des représentations. De plus, nous pouvons estimer que la probabilité qu'il ait une expérience de formation à distance soit faible. Ceci signifie que l'apprenant s'attend à retrouver une situation proche de celle d'un enseignement en présentiel plutôt de type ex cathedra.

Le choc des représentations préalables aux représentations d'un nouvel enseignement est une phase délicate. Effectivement, la tendance chez les apprenants est d'avoir. une préférence à garder ses représentations préalables comme plus exactes que les nouvelles enseignées ou pratiquées. Dans notre contexte, cela se traduit par un risque de rejet du système d'enseignement à distance. En pratique, ce rejet se concrétise par l'abandon.

Pour ces raisons, le choix a été de tenter d'arriver à un enseignement à distance qui soit un enseignement virtuellement en présentiel. Avec la technologie, il est aujourd'hui possible d'atténuer la distance physique en utilisant par exemple la visioconférence ou d'atténuer la distance temporelle par l'organisation de contact par courrier électronique. Néanmoins, pour cette recherche, la volonté a été de garder un système de formation à distance "pure" c'est-à-dire avec une rupture de la communication, avec une séparation des actes d'enseignement et d'apprentissage. Il s'agit d'une option motivée par la volonté de laisser à l'apprenant la liberté de suivre l'enseignement dans les lieux et au moment qu'il choisit.

Dès lors, nous nous sommes tourné vers l'enregistrement filmé d'un cours. La formule est simple, elle est donc peu onéreuse. Elle permet d'éviter un grand stockage voire tout stockage. La distribution est également aisée. Cette formule répond aux contraintes économiques. Elle est manifestement à distance mais en même temps elle se

Marcel Crochet, recteur de l'Université catholique de Louvain, dans la revue "Louvain" n' 108 , mai 2000. 
rapproche de l'enseignement classique et donc des représentations moyennes des apprenants adultes. Il reste la difficulté de la rupture de la communication. En d'autres termes, la question est de savoir comment tenter de créer un réseau de communication qui place l'apprenant à distance dans une situation où il se perçoit comme étant virtuellement dans un enseignement en présentiel tout en étant réellement dans un enseignement à distance.

Le raisonnement a été de tenter d'impliquer l'apprenant à distance de la même façon qu'un étudiant à un cours en présentiel. Dans ce dessein, l'implication a été réfléchie par le biais de l'identification. L'idée contient deux aspects. D'une part, plus l'apprenant à distance se perçoit comme les apprenants filmés, plus il travaillerait par procuration et plus l'enseignement lui serait bénéfique pour son apprentissage. D'autre part, plus l'apprenant à distance se perçoit comme les apprenants filmés, plus il se sentirait appartenir au groupe fictif composé des étudiants filmés et lui, moins il percevrait sa situation d'isolement. Dans cette conception, l'étudiant filmé doit être un support positif à l'apprentissage de l'apprenant à distance.

Pour créer le processus d'identification, la similitude entre la ou les personnes filmées et celle qui regarde ou la ressemblance positive c'est-à-dire une similitude améliorée sont les moyens les plus régulièrement cités dans la littérature scientifique.

\section{La mise en ouvre de l'expérimentation}

Une préexpérimentation fut réalisée. Le but de cette première expérience fut de tester l'expérience en elle-même et d'avoir une première esquisse du bien fondé de l'hypothèse. Avec les enseignements acquis dans la préexpérience, l'expérience a été construite avec trois documents audiovisuels réalisés au départ de deux enregistrements. Le thème du cours était l'énonciation audiovisuelle.

L'enseignant et les étudiants filmés étaient des volontaires. Le premier enseigne à l'Université catholique de Louvain au département de communication; les seconds en étaient des étudiants. Avant l'enregistrement du cours, les objectifs poursuivis leur ont été expliqués.

Un premier enregistrement a filmé l'enseignant donnant son cours devant quatre étudiants. Après une introduction donnée par un exposé ex cathedra, l'enseignant a montré trois spots publicitaires 
axés sur la prévention du SIDA. Ensuite, l'enseignant a analysé les spots avec les étudiants. Il s'agit d'un travail collectif dirigé par l'enseignant. Enfin, l'enseignant a synthétisé le travail accompli par le groupe. Aucun scénario n'avait été défini préalablement mais avant l'enregistrement, l'instruction donnée au professeur était de donner un cours susceptible d'être compris par tous les étudiants universitaires quelle que soit leur année et leur discipline, et de susciter des interactions entre lui et les étudiants. Les étudiants avaient, dans le cadre de leur formation, déjà vu la matière du cours. Il leur était donc aisé d'intervenir dans l'analyse des spots publicitaires.

Le second enregistrement a filmé l'enseignant donnant seul son cours devant la caméra ${ }^{1}$. L'enseignant a évidemment effectué seul l'analyse des spots publicitaires. Néanmoins dans son discours, il a tenté d'interpeller ses étudiants de l'autre côté de l'écran en utilisant des formes oratoires du type : "Et que pourriez-vous me répondre ?", "Que penser de la séquence...?", "comme vous me diriez", "vous dégagez donc que", "vous allez dès lors me répondre"... A toutes ces interpellations, l'enseignant a répondu. Nous pouvons parler d'une forme d'interactions simulées de type oratoire.

Des deux enregistrements, trois montages ont été réalisés. Le document 1 ne reprend que le second enregistrement. Les documents 2 et 3 ont été montés au départ des deux enregistrements. Les trois montages ont été conçus en veillant à minimiser les différences, excepté celles liées aux interactions et à la présence des étudiants filmés. Deux faits sont à noter. Lors du premier enregistrement, l'enseignant est plus long dans son introduction que lors du second enregistrement. Chacune des deux introductions était une intervention ex cathedra de l'enseignant. La différence de temps résulte de plus de redondances et d'explications données par le professeur lors du premier enregistrement parce qu'il y avait la présence d'étudiants. Le second fait intéressant est de constater que l'enseignant seul analyse les spots publicitaires en $18^{\prime} 50^{\prime \prime}$ contre 32 ' avec les étudiants soit $70 \%$ de temps en plus.

Le tableau ci-dessous reprend les trois documents audiovisuels en indiquant par partie, l'origine de l'enregistrement. Ceci permet de

1 Pour s'assurer du même contenu, il fut impératif d'enregistrer en premier lieu le document filmant l'enseignant donnant son cours devant les étudiants. Seul devant la caméra, l'enseignant a pu aisément reprendre une matière identique à celle du premier enregistrement. 
voir les différences et les similitudes entre les documents audiovisuels.

\begin{tabular}{|c|c|c|c|}
\hline Plan du cours & $\begin{array}{c}\text { Document } 1 \\
\text { Enseignant seul }\end{array}$ & $\begin{array}{c}\text { Document } 2 \\
\text { Enseignant avec } \\
\text { des étudiants } \\
\text { sans interaction }\end{array}$ & $\begin{array}{c}\text { Document } 3 \\
\text { Enseignant avec des } \\
\text { étudiants avec des } \\
\text { interactions }\end{array}$ \\
\hline Introduction $\left(9^{\prime} 40^{\prime \prime}\right)$ & \multicolumn{3}{|c|}{$\begin{array}{l}\text { Deuxième enregistrement, enseignant seul } \\
\text { devant la caméra } \\
\qquad \begin{array}{c}\text { +vues d'étudiants sans interaction } \\
\text { premier enregistrement : enseignant et } \\
\text { étudiants avec interactions }\end{array}\end{array}$} \\
\hline & \multicolumn{3}{|c|}{ Visionnement des spots publicitaires ( $\left(20^{\prime \prime}\right)$} \\
\hline $\begin{array}{l}\text { Analyse des spots } \\
\text { et conclusion }\end{array}$ & $\begin{array}{r}\text { Deuxième enreg } \\
\text { seul dev }\end{array}$ & $\begin{array}{l}\text { ement, enseignant } \\
\text { la caméra } \\
\text { + vues d'étudiants } \\
\text { sans interaction } \\
\text { premier } \\
\text { enregistrement : } \\
\text { enseignant et } \\
\text { étudiants avec } \\
\text { interactions } \\
\text { - - - }\end{array}$ & $\begin{array}{c}\text { Premier } \\
\text { enregistrement, } \\
\text { enseignant avec } \\
\text { des étudiants et } \\
\text { des interactions } \\
\text { (32'00") }\end{array}$ \\
\hline Durée totale & \multicolumn{2}{|c|}{$30^{\prime} 50^{\prime \prime}$} & $44^{\prime} 00^{\prime \prime}$ \\
\hline & $\begin{array}{c}\text { Groupe 1 } \\
\text { d'étudiants à } \\
\text { distance testés }\end{array}$ & $\begin{array}{c}\text { Groupe } 2 \\
\text { d'étudiants à } \\
\text { distance testés }\end{array}$ & $\begin{array}{c}\text { Groupe } 3 \\
\text { d'étudiants à } \\
\text { distance testés }\end{array}$ \\
\hline
\end{tabular}

Ces trois documents audiovisuels ont été les outils de base de l'expérience.

Vingt-huit étudiants ont participé à l'ensemble de l'expérience comme apprenants à distance. Ils étaient tous volontaires. Neuf étaient soit diplômés (7), soit étudiants en fin d'un cycle universitaire (2) dans le domaine de l'économie. Seize étaient étudiants en première année d'université ( 7 en histoire et 9 en philologie romane) et trois en deuxième année d'université en philologie romane. Notons que les vingt-huit étudiants testés étaient vingt femmes et huit hommes répar- 
tis en onze personnes de moins de vingt ans, treize entre vingt et vingt-quatre ans, un de vingt-huit ans, un de trente ans, un de trentesept ans et un de quarante-six ans.

Trois groupes d'étudiants à distance ont été constitués. Pour la composition des groupes, la répartition des étudiants d'une même année académique a été effectuée de façon équivalente dans les trois groupes. Ces proportions respectées, les étudiants de même année académique ont été répartis aléatoirement dans un des trois groupes.

Les apprenants à distance se distinguent par rapport au type de document audiovisuel qu'ils ont visionné. Ainsi, les groupes se définissent comme suit :

gr1 $=$ ceux qui visionnent le document audiovisuel avec l'enseignant seul, sans interaction excepté des interactions fictives sous la forme oratoire (document 1 )

gr2 $=$ ceux qui visionnent le document audiovisuel avec l'enseignant et 4 étudiants mais sans interaction excepté des interactions fictives sous la forme oratoire (document 2)

gr3 $=$ ceux qui visionnent le document audiovisuel avec l'enseignant et 4 étudiants et avec des interactions enseignant / étudiants et des interactions fictives sous la forme oratoire (document 3 )

Le dispositif a été conçu en trois phases :

Première phase :

1) Observation $\mathrm{O}_{0}$ : définition du type de l'étudiant : mesure (par test) des besoins des étudiants (encadrement, appartenance, interaction)

2) Observation $\mathrm{O}_{1}$ : prétest de connaissances sur la matière du cours

Deuxième phase (au moins une semaine après la première phase)

3) Visionnement du cours audiovisuel (observation de l'activité de l'étudiant)

4) Observation $\mathrm{O}_{2}$ : test de connaissances sur la matière du cours

5) Interview dirigée des étudiants

Troisième phase (huit semaines après la deuxième phase)

6) Observation $\mathrm{O}_{3}$ : test différé de connaissances sur la matière du cours

7) Observation O0bis :vérification du type de l'étudiant : mesure (par test) des besoins des étudiants (encadrement, appartenance, interaction) 


\section{Les résultats principaux de l'expérimentation}

Trois grandes options ont été suivies pour analyser les résultats. Les deux premières ont consisté à analyser l'évolution des apprentissages des étudiants à distance d'une part sur base du document audiovisuel visionné et d'autre part sur base de la définition de profils des apprenants à distance. Soulignons qu'aucun résultat statistiquement significatif ne s'est dégagé. La tendance à long terme la plus marquante ne peut que satisfaire les institutions universitaires comme celle de Louvain : plus l'apprenant a une expérience académique importante réussie, plus il apprend.

La troisième approche fut sans conteste la plus intéressante. Il s'agit de l'analyse des perceptions des apprenants à distance sur base des interviews. Le système des interviews fut celui de l'entonnoir en vue d'arriver à ce que l'étudiant à distance exprime sa perception de la présence ou de la non-présence d'étudiants filmés.

Sur base des sentiments exprimés par les étudiants testés, nous pouvons établir une brève synthèse en huit points reprenant les éléments essentiels dégagés de l'analyse des interviews.

1. Il ressort que la variable la plus importante qui influence les perceptions du cours audiovisuel est le type de film visionné.

2. Le degré d'intérêt de la présence des étudiants filmés pour l'apprenant à distance est lié essentiellement aux interactions réelles.

3. Les documents où les étudiants filmés interviennent sont appréciés pour l'expression d'autres avis, le dialogue facilitateur d'accès à la matière et à la prise de notes, la guidance de l'enseignant filmé, le stimulant et/ou la sortie de l'isolement. Il est à noter que l'attention est pour certains stimulée et pour d'autres, c'est l'inverse.

4. Si les étudiants filmés n'interviennent pas, ils ne sont pas appréciés ; aucun effet n'est perçu même si pour plusieurs étudiants testés, les étudiants filmés silencieux précisent le cadre.

5. Sept des dix étudiants ayant visionné le document avec les interactions parlent d'identification, neuf d'entre eux évoquent au moins soit l'identification, soit l'implication, soit l'appartenance au groupe.

6. L'implication n'est pas évoquée par les étudiants ayant visionné le document avec l'enseignant seul. L'interaction suscite plus l'implication que la seule présence des étudiants filmés. 
7. Le sentiment d'appartenance ou non à un groupe fictif ne semble pas varier de façon significative entre les trois groupes. Dans les différents groupes, les étudiants testés considèrent plutôt que l'enseignant s'adresse à une classe.

8. La similarité avec un cours en auditoire est perçue avec un degré plus élevé si le cours filmé ne présente que l'enseignant avec une méthodologie pédagogique du type ex cathedra.

\section{La confrontation des résultats de l'expérimentation et de l'hypothèse}

Globalement, très peu d'effets se sont dégagés de la présence d'étudiants filmés si ceux-ci n'interagissent pas. Par contre, les interactions ont un impact sur l'appréciation du cours, l'approche de la matière, les processus d'identification et d'implication. La variable majeure pour classer les étudiants testés est donc la présence ou non d'interactions réelles.

Nous pouvons considérer que les séquences d'interactions réelles entre l'enseignant et les étudiants filmés dans le document audiovisuel suscitent une implication positive de l'apprenant à distance. Il y a donc des relations communicationnelles, (soit un réseau de communication), qui se créent entre l'apprenant à distance et les acteurs filmés.

Les interactions réelles reprises dans le document audiovisuel sont des moyens pédagogiques pour créer une activation de l'apprenant à distance. Elles aident à transformer l'apprenant à distance "télé-spectateur" en un apprenant à distance "télé-acteur".

Notons que les étudiants qui ont exprimé une activation plus grande lors du cours sont majoritairement du type "bons" étudiants du point de vue des résultats académiques.

De ces éléments, on peut tirer l'hypothèse générale suivante : les interactions dans le document audiovisuel de formation créent un système communicationnel favorable à l'activation cognitive de l'apprenant à distance.

Les "non-intervenants" c'est-à-dire les étudiants filmés qui ne disent rien, ne sont que des parties du décor qui apportent une information mais qui peuvent être un parasite à la communication. L'apprenant à distance est dans une situation de spectateur d'une relation de communication qui est conçue pour s'adresser à lui. Quand des participants à cette relation de communication ne jouent qu'un 
rôle de spectateurs, il y a une forme de malentendu ressenti par l'apprenant à distance. Ce malentendu devient un parasite dans la communication parce que l'apprenant à distance qui n'accepte pas cette situation, ne peut pas la modifier. Il reste donc dans une situation d'inconfort. Dans l'expérience, entre le discours de l'enseignant et le décor qu'est l'étudiant filmé qui n'intervient pas, il y a pour l'apprenant à distance une discordance. Les interactions orales stimulatives sont "anormales" par rapport au contexte et elles suscitent le malaise.

L'inclusion ou non dans le réseau de communication final de l'enseignant et des étudiants filmés est liée à l'activité des acteurs. Les interactions réelles assurent une participation au réseau de communication final. L'importance ou le nombre d'interactions assurent le degré d'implication dans le réseau de communication.

La figure ci-dessous reprend toutes les situations avec l'ensemble des acteurs dans le même schéma : document audiovisuel, apprenant à distance, enseignant, étudiants intervenant et étudiants n'intervenant pas. Elle met en évidence la construction du réseau de communication final.

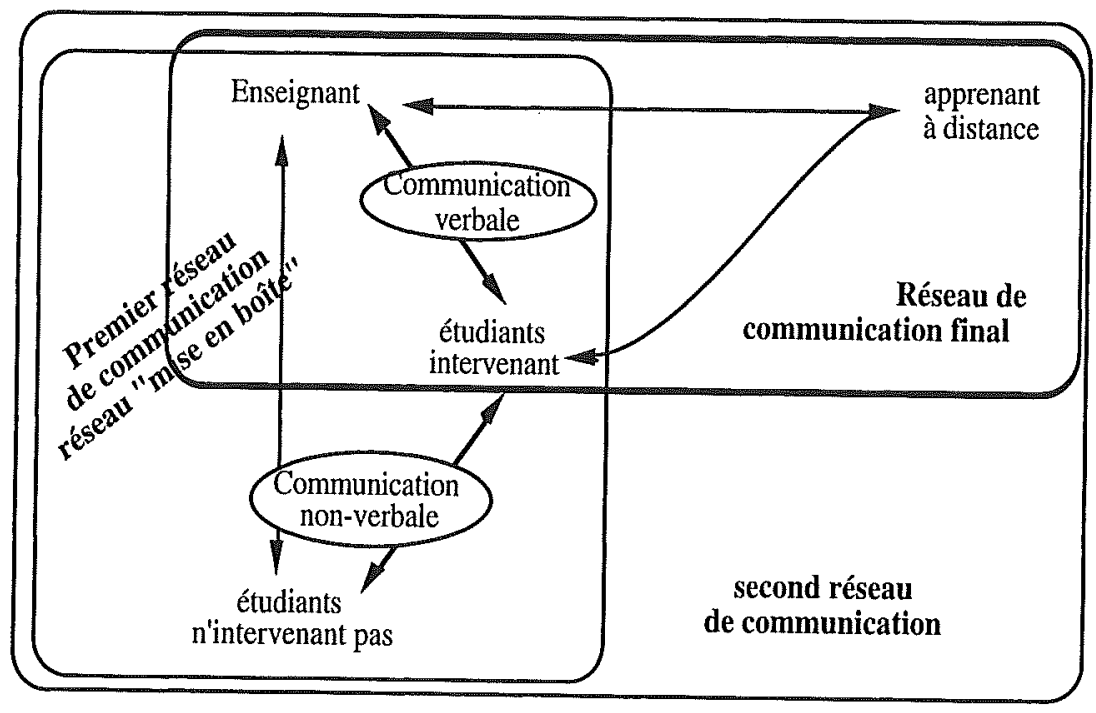

Réseaux de communication qui s'établissent dans le cadre d'un cours audiovisuel filmant des étudiants qui interagissent ou qui n'interagissent pas. 


\section{Des pistes de réflexions futures}

Traçons quelques pistes de recherches futures en nous limitant aux thèmes qui apparaissent comme essentiels. Dans tout enseignement, l'activité de l'apprenant est un facteur de grande importance pour la réussite de la formation. La formation à distance et en particulier les documents audiovisuels de formation sont confrontés à cette même problématique.

Une prolongation de ce travail de recherche pourrait être l'examen des effets des différents types d'interactions, avec ou sans rupture de la communication, sur l'activation et sur l'apprentissage des apprenants intervenant dans les interactions et des apprenants spectateurs des interactions. A priori, l'apprenant intervenant dans l'interaction devrait bénéficier d'un meilleur apprentissage à court et à long terme. Cette recherche, sur base d'une typologie des interactions, pourrait dès lors analyser les conditions et les types d'interactions qui placent l'apprenant à distance dans la situation la plus profitable à son apprentissage. Dans ces conditions devrait être reprise l'étude des moyens technologiques les plus appropriés. La définition des conditions devrait tenir compte des différents profils d'apprentissage.

Ces aspects peuvent également être étudiés par rapport au problème des abandons en formation à distance.

Sur base de la recherche, considérer les interactions non-verbales du cours filmé comme sans effet apparaît logique mais scientifiquement quelque peu téméraire. Une expérimentation nouvelle avec un dispositif permettant de tester les effets de la communication nonverbale du cours filmé sur la construction du réseau communicationnel d'ensemble devrait être réalisée.

\section{Et pour clore}

Manifestement, nos résultats s'inscrivent dans des courants actuels d'idées et de réflexions communicationnelles et pédagogiques.

Les interactions constituent un système. Nous avons construit notre réflexion communicationnelle sur la base d'un réseau de communication composé de relations communicationnelles qui constituent un système. Notre recherche s'inscrit dans la logique de la 
communication développée dans le cadre de l'étude de la structure de l'interaction humaine de l'école de Palo Alto'.

Dans notre expérience, nous étions dans une situation d'interactions entre l'apprenant et le support contenant les connaissances. Les interactions sont essentielles dans la création des relations communicationnelles. Dans le cadre d'un cours, les interactions sont aussi un élément de grande importance pour l'activation de l'apprenant pendant le cours. En pédagogie, plusieurs auteurs placent l'interaction comme un aspect fondamental de leurs recherches. Nos résultats et les différentes conclusions que nous avons tirées, nous placent aussi dans ce courant d'idées pédagogiques.

Nous avons travaillé des outils pédagogiques en formation à distance. Peuvent-ils aussi être des supports pour la formation en présentiel ? Dans l'enseignement en présentiel, l'enseignant peut se mettre en situation de formation à distance. Ainsi, afin de stimuler ses étudiants à travailler leurs capacités cognitives et métacognitives, l'enseignant peut virtuellement se faire absent de la classe pour laisser ses élèves travailler seuls, indépendamment de lui, avec des outils pédagogiques ${ }^{2}$.

Nous pensons donc que des pratiques et des outils pédagogiques d'un type d'enseignement peuvent être transférés à l'autre type sachant qu'il est nécessaire de construire un cours en tenant compte de ses objectifs, de garder la variété d'enseignement dans un cours et d'adapter un cours à son public étudiant et à son contexte. Il n'y a donc pas lieu de cloisonner les deux types de formation.

Pour clore cet article, rappelons que l'option prise a été un enseignement en présentiel virtuel. Mais la formation à distance est une formation avec une distance entre l'enseignant et ses étudiants. Le monde du virtuel, de plus en plus présent dans la vie réelle, est susceptible, par la communication qu'il propose, de diminuer virtuellement la distance réelle et de susciter un sentiment réel de création d'une relation qui, en réalité, est virtuelle.

\footnotetext{
1 Nous nous référons entre autres à "Une logique de la communication" de P. Watzlawick, J. Helmick Beavin, Don D. Jackson, Paris, Éd. du Seuil, 1972.

2 Le bon enseignant en présentiel a la capacité de se taire, il n'a pas peur des silences.
} 